\title{
Expressions of ZNF436, $\beta$-catenin, EGFR, and CMTM5 in breast cancer and their clinical significances
}

\author{
Zhi Chen, ${ }^{1 *}$ Na Cui, ${ }^{2}$ Ji-sen Zhao, ${ }^{1}$ Jian-fei Wu, ${ }^{1}$ Fang Ma, ${ }^{1}$ Cong Li,1 Xian-yi Liu ${ }^{1 *}$ \\ ${ }^{1}$ Department of Hepatobiliary Surgery, Affiliated Hospital of Hebei University, Baoding, Hebei \\ ${ }^{2}$ Department of ICU, Affiliated Hospital of Hebei University, Baoding, Hebei \\ ${ }^{3}$ Department of Breast Surgery, Affiliated Hospital of Hebei University, Baoding, Hebei, China \\ *These authors contributed equally
}

As the leading malignancy among women, breast cancer is a serious threat to the life and health of women. In this context, it is of particular importance that a proper therapeutic target be identified for breast cancer treatment. We collected the pathological tissues of 80 patients, with the view to discovering appropriate molecular targets for the treatment of breast cancer, this paper analyzes the expressions of ZNF436, $\beta$-catenin, EGFR and CMTM5 in breast cancer tissues, as well as their correlations with breast cancer in combination with the clinicopathologic characteristics of studied patients. Immunohistochemistry was utilized to detect the expression levels of ZNF436, $\beta$-catenin, EGFR and CMTM5 in cancerous and paracancerous tissues of breast cancer patients. The expression levels of ZNF436, $\beta$-Catenin and EGFR in breast cancer tissues were significantly greater than those in paracancerous tissues in this study $(\mathrm{p}<0.05)$, while CMTM5 was highly expressed in paracancerous tissues $(\mathrm{p}<0.05)$. Additionally, the correlation of the expressions of such indicators with the staging, differentiation and lymphatic metastasis of breast cancer, were also found to be statistically significant at the level $p<0.05$. The different expression levels of ZNF436, $\beta$-catenin, EGFR and CMTM5 in breast cancer and paracancerous tissues open up the possibility of utilizing them as molecular markers for breast cancer. These findings provide a theoretical basis for targeted molecular therapies for breast cancer, and hence carry a significant practical significance.

Key words: Breast cancer; ZNF436; $\beta$-catenin; EGFR; CMTM5; immunohistochemistry.

Correspondence: Xian-yi Liu, Department of Breast Surgery, Affiliated Hospital of Hebei University, Baoding, Hebei 071000, China. E-mail: nzix32@163.com

Contributions: ZC, XL, carried out the studies, participated in collecting the data, drafted the manuscript and were responsible and accountable for the accuracy and integrity of the work; NC, JZ, JW, performed the statistical analysis and participated in the study design; FM, CL, participated in the acquisition, analysis, or interpretation of the data and drafted the manuscript. All the authors have read and approved the final version of the manuscript and agreed to be accountable for all aspects of the work.

Funding: This research received no specific grant from any funding agency in the public, commercial, or notfor-profit sectors.

Availability of data and materials: All data generated or analyzed during this study are included in this published article.

Ethical Approval: The study was approved by the Institutional Ethics Committee of Affiliated Hospital of Hebei University, and written informed consent was obtained from all participants.

Informed consent: There are no human subjects in this article, and informed consent is not applicable. 


\section{Introduction}

Breast cancer is deemed as one of the life- and health-threatening malignancies among women. Since the 1970s, the incidence of breast cancer has been on the rise. According to relevant statistical data, the incidence of breast cancer surpasses those of other malignancies among women, with the incidence in urban areas eclipses that in rural areas. ${ }^{1}$ The incidence of breast cancer also tends to firstly rise before dropping with age. In other words, younger women have lower chances of getting breast cancer, whereas the incidence peaks among the middle-aged women. ${ }^{2}$ In response to this issue, over the years, scholars have attempted to make relevant investigations from a molecular perspective, explored possible pathogenic mechanisms, and strived to increase the precision of targeted therapies, all with the ultimate view to conquering breast cancer as soon as possible. ${ }^{3}$

Studies have found that zinc finger protein 436 (ZNF436), $\beta$ catenin, epidermal growth factor receptor (EGFR), and CKLF-like MARVEL transmembrane domain-containing protein 5 (CMTM5) are differentially expressed in different tumors. For instance, ZNF436 has been verified as a cancer-promoting gene that is highly expressed in tumor-associated cells, one of which are glioma cells. As pointed out by Shang et al., ${ }^{4}$ it is possible to inhibit the proliferation of glioma cells by silencing the genetic expressions of ZNF436. $\beta$-catenin is also found to mostly participate in embryonic development and tissue homeostasis among various other diseases via the $\mathrm{Wnt} / \beta$-catenin signaling pathway. Once this pathway is abnormally activated, $\beta$-catenin may excessively accumulate in cells, which then promotes the transcription of oncogene, and in turn making $\beta$-catenin responsible for the incidences and progressions of head and neck tumors, ovarian cancers, pancreatic cancers, and lung cancers. ${ }^{5-7}$ EGFR, which is widely distributed on cell surfaces, not only plays a critical role in the growth, proliferation and differentiation of cells on the one hand; but may also trigger the formation of tumors through mutation or over-expression on the other hand. For this reason, investigating its influence on tumor occurrence and progression is of particular importance. ${ }^{8,9}$ Moreover, EGFR is also critical to self-healing in the human body, which elevates its significance in biological practices. Last but not least, CMTM5, while being poorly expressed in multiple tumor cells, its overexpression can apparently promote tumor cells apoptosis as well as inhibit their proliferation and migration. ${ }^{10-13}$

Although the relationships of these genes with other tumors have become increasingly clear with every research done, and although these genes have been known to offer a range of promotional or inhibitive effects on different tumors, relevant information on breast cancer has not been sufficiently investigated, while their correlations with the patients' clinical characteristics are also yet to be clarified. ${ }^{14-17}$ In light of this background, this study collected specimens from patients during clinical practices to test the levels of expression of these genes and analyze how the changes in their expression levels would correlate with the patients' age, sex, tumor size, lymphatic metastasis, TNM staging, etc., providing some theoretical grounds for a deeper understanding into the pathogenesis of and development of cure for breast cancer.

\section{Materials and Methods}

\section{Sample sources}

With the consent of themselves and their families, 80 pairs of specimens were taken from patients pathologically diagnosed with breast cancer from October 2018 to April 2019 in the Affiliated Hospital of Hebei University. Each of the specimen pair include cancerous and paracancerous tissues, with the latter taken from about $5 \mathrm{~cm}$ away from where the former was extracted.

The corresponding inclusion criteria are as described in the following: i) the breast cancer should be identified as primary breast cancer by a postoperative pathological examination; ii) no history of any preoperative treatment, such as chemoradiotherapy; iii) the medical history and follow-up information are all complete; and iv) no other neoplastic diseases or autoimmune diseases. The relevant exclusion criteria are as follows: i) have undergone relevant pre-operative treatment(s); ii) have a history of malignant tumors; and iii) follow-up information is incomplete.

All the 80 patients enrolled were newly diagnosed, and their age ranges between $33 \sim 72$, averaging at 55.25 years. Based on the classification stipulated in the AJCC Cancer Staging Manual $\left(7^{\text {th }}\right.$ edition), 30 patients were identified as stages I+II patients, while the remaining 50 were in stages III+IV. Their cancerous and precancerous tissues were obtained through surgical excision, and then preserved in a $4 \%$ paraformaldehyde solution for immunohistochemistry (IHC) later on.

\section{Main instrument and reagents}

The main instruments and reagents used in this research are as listed in Tables 1 and 2.

\section{Experimental methods}

The cancer tissues and adjacent tissues obtained from the surgery were fixed in $4 \%$ paraformaldehyde for $48 \mathrm{~h}$. To begin with, the fixed tissue samples were subject to dehydration and waxing, and tissue blocks were placed in the embedding machine for embedding and then in the freezing machine, where they were frozen. After that, the paraffin blocks were cut into $3 \sim 4 \mu \mathrm{m}$ slices, before being spread and roasted. Paraffin in the roasted slices was removed and replaced with water to perform antigen retrieval. After that, the samples were incubated in 3\% hydrogen peroxide for $25 \mathrm{~min}$ and then sealed with the serum. Subsequently, the ZNF436, $\beta$-catenin, EGFR, and CMTM5 were added into their respective primary antibody solutions (diluted at 1:200, AF0427,

Table 1. Main experimental instruments.

\begin{tabular}{lccc}
\hline Instrument & Model No. & Manufacturer & Place of origin \\
Paraffin slicing machine & RM2235 & Leica & Germany \\
Electro-thermostatic blast oven & QH01-9030A & Jing Hong Laboratory Instrument & Shanghai \\
\hline Electro-heating standing-temperature cultivator & DH36001B & Tianjin Taisite & Tianjin \\
Ultra Purified Water System & NW10LVF & Heal Force & China \\
\hline Microscope & BX53 & OLUMPUS & Japan \\
Camera system of the microscope & DP73 & OLUMPUS & Japan \\
\hline
\end{tabular}


Affinity, Beijing, China; diluted at 1:100, WL0962a, Wanleibio, Shenyang, China; diluted at 1:100, WL0682a, Wanleibio, Shenyang; diluted at 1:50, D160395, Sangon Biotech, Shanghai) for incubation overnight at $4{ }^{\circ} \mathrm{C}$. Then, they were rinsed with PBS before being incubated in the secondary antibody solution (diluted at 1:5000,\#31460, ThermoFisher, Waltham, MA, USA) for $50 \mathrm{~min}$ at room temperature. Afterwards, they were washed with PBS and added with DAB color developing solution drop-by-drop until the tissues were uniformly covered. The slices were then counterstained for 2 min using hematoxylin (G1004-100, Servicebio, Wuhan, China), before they were dehydrated, applied with a mounting medium, and mounted with a coverslip. Set the microscope magnification to 400 times, and randomly select the brownyellow area as the field of vision to be observed. At this point, the prepared slices were ready to be clipped on the microscope's stage to be observed. It could be seen that the target protein turned brownish yellow due to the staining. For each slice, three different areas were captured and saved. Image-Pro Plus was used to carry out photodensitometry for each picture, and the average optical density was calculated from the three pictures of the same slide.

\section{Statistical analysis}

Data processing was completed in SPSS 20.0, and all the results are expressed in mean \pm standard deviation $(\mathrm{M} \pm \mathrm{SD})$. The enumeration data were processed via the Chi-square test. This study assumes $\mathrm{p}<0.05$ as the threshold for statistical significance $(\mathrm{p}<0.05, \mathrm{p}<0.01$, or $\mathrm{p}<0.001)$. The relevant graphs were generated in GraphPad Prism 8.0.

\section{Results}

\section{Expression levels of ZNF436, $\beta$-catenin, EGFR and CMTM5 in breast cancer}

Figure 1 shows the examples of the immunostaining for ZNF436 (Figure 1A, Supplementary Figure 1), $\beta$-catenin (Figure 1B, Supplementary Figure 2), EGFR (Figure 1C, Supplementary Figure 3) and CMTM5 (Figure 1D, Supplementary Figure 4) in breast cancer and paracancerous tissues. The average optical density for ZNF436 from the 80 cancerous tissues was 0.0146 and its expression level was 9.125 times greater than that of paracancerous tissues (0.0016). It was highly expressed in the cancerous tissues, showing statistically significant differences $(p<0.05)$. The average optical density for $\beta$-catenin among the cancerous tissues was 0.0285 , which was 1.84 times larger than that in paracancerous tissues (0.0155), showing statistically significant differences at the level of $p<0.05$. Likewise, EGFR was also highly expressed in the cancerous tissues and its expression level was 1.2 times greater than that in paracancerous tissues, showing statistically significant differences $(\mathrm{p}<0.05)$. On the contrary, CMTM5 was more highly expressed in the paracancerous tissues at a level of 1.3 times greater than that in the cancerous tissues, showing statistically significance differences as well $(\mathrm{p}<0.05)$.

\section{Variations in expression levels of ZNF436, $\beta$-catenin, EGFR and CMTM5 in breast cancer, and analysis on their correlations to clinicopathological characteristics}

By combining clinicopathological information of patients, an analysis was made on the correlations of the expression levels of these proteins with respect to the clinicopathological characteristics. As indicated by the analysis results, their expression variations were significantly correlated with the clinical staging, differentiation, and lymphatic metastasis of patients $(p<0.05)$ but showed no correlation with the patients' age and tumor size. The data have been presented in detail in Tables 3 and 4 .

\section{Expressions of tissue repair related protein EGFR and analysis on their correlations to clinicopathological characteristics of patients}

In addition to analyzing the variations in expressions of EGFR in tissues of patients with breast cancer, their correlations with clinicopathological characteristics of patients are also analyzed. The corresponding results are presented in Table 5. As can be observed, EGFR expression levels were closely correlated with clinical staging, tumor differentiation and lymphatic metastasis of the patients, showing statistical significance at the level of $\mathrm{p}<0.05$.

\section{Discussions}

Breast cancer is induced by the uncontrolled proliferation of mammary epithelial cells under the actions of carcinogenic factors. Early onset of breast cancer is manifested in breast lumps and nipple discharge, etc. in most cases. Because such symptoms are rather inconspicuous, it is much likely for them to be overlooked. Over $70 \%$ patients have reached the advanced stage when seeking medical advice. At the advanced stage, lymphatic metastasis has occurred in most cancers, often accompanied with multiple-organ pathology, leading to great difficulty in curing and high mortality. Without a doubt, breast cancer is a serious threat to the lives and wellbeing of patients. ${ }^{18}$ How to implement early prevention, early diagnosis and early treatment of breast cancer seem to be especially important.

Table 2. Main experimental reagents.

\begin{tabular}{|c|c|c|c|}
\hline Reagent & Article No. & Manufacturer & Place of origin \\
\hline ZNF436 & AF0427 & Affinity & China \\
\hline$\beta$-catenin & WL0962a & Wanleibio & China \\
\hline EGFR & WL0682a & Wanleibio & China \\
\hline CMTM5 & D160395 & Sangon Biotech & China \\
\hline HRP-labeled goat anti-rabbit IgG & $\# 31460$ & thermoFisher & the US \\
\hline Absolute ethyl alcohol & 10009218 & Sinopharm & China \\
\hline Hematoxylin & H8070 & Solarbio & China \\
\hline Goat serum & SL038 & Solarbio & China \\
\hline DAB colour-developing reagent & DA1010 & Solarbio & China \\
\hline Hydrogen peroxide & 10011218 & Sinopharm & China \\
\hline
\end{tabular}




\section{A Cancer tissue}

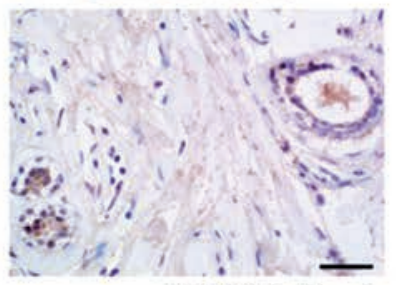

ZNF436 Poorly'differentiated
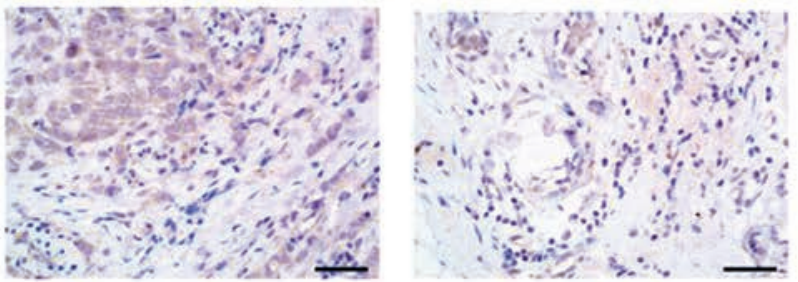

ZNF436 Moderately differentiated
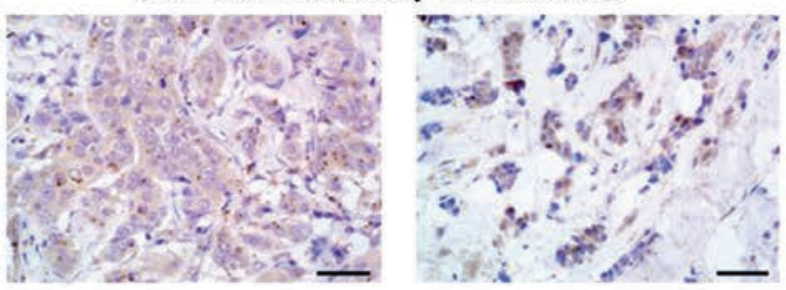

ZNF436 Highly differentiated

\section{Cancer tissue}

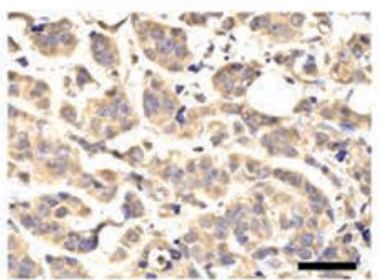

EGFR Poorly differentiated
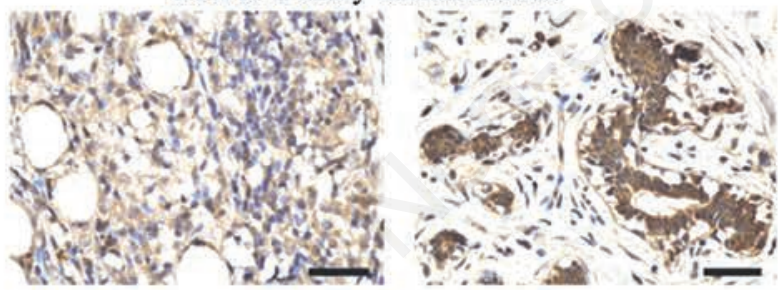

EGFR Moderately differentiated
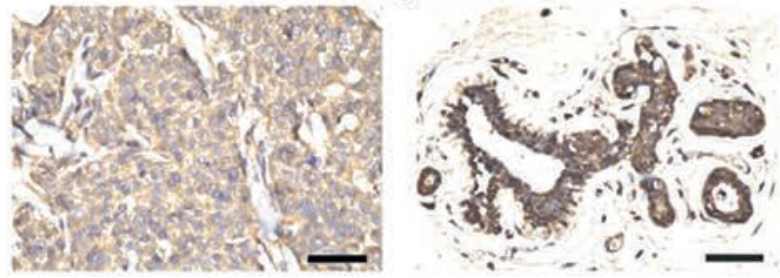

EGFR Highly differentiated
B Cancer tissue

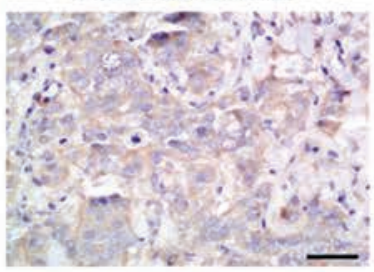

Paracancerous tissue

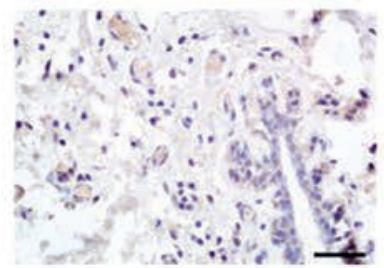

$\beta$-catenin Poorly differentiated
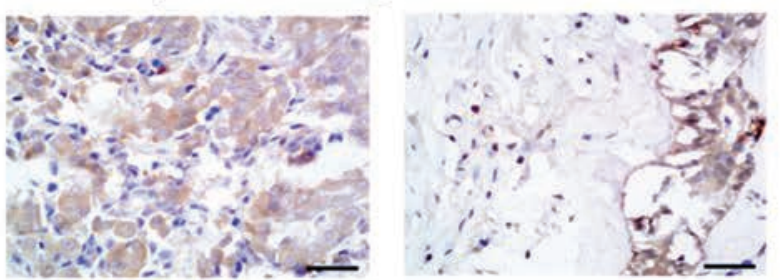

$\beta$-catenin Moderately differentiated

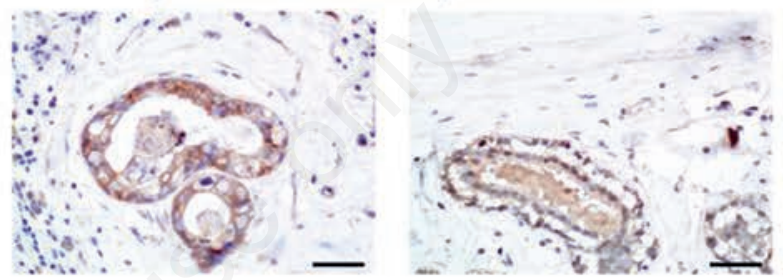

$\beta$-catenin Highly differentiated

\section{Cancer tissue}

\section{Paracancerous}
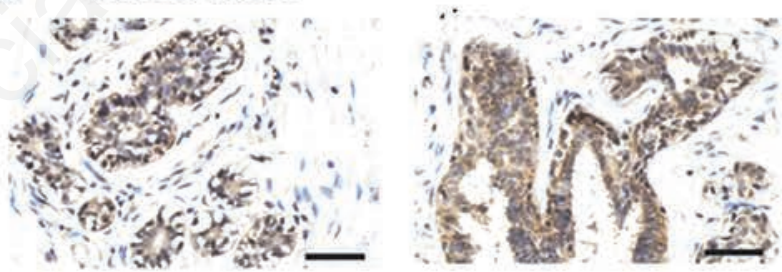

CMTM5 Poorly differentiated
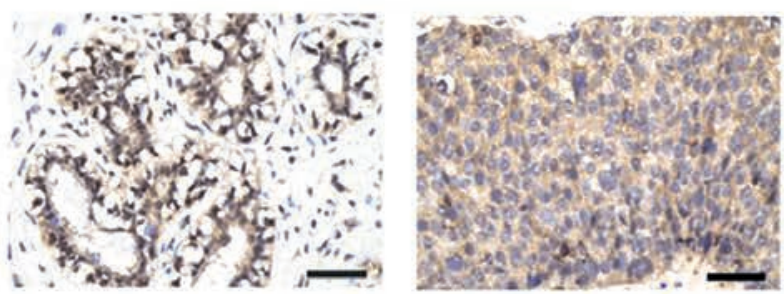

CMTM5 Moderately differentiated
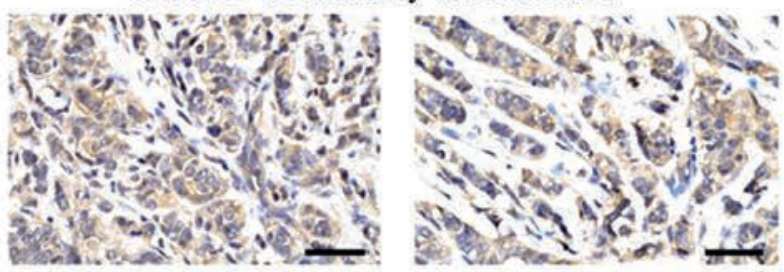

CMTM5 Highly differentiated

Figure 1. Expression levels of ZNF436, $\beta$-catenin, EGFR and CMTM5 in breast cancer and paracancerous tissues. The expression levels of ZNF436, $\beta$-catenin and EGFR in breast cancer tissues were significantly higher than those in adjacent tissues (p<0.05); CMTM5 was highly expressed in adjacent tissues $(\mathrm{p}<0.05)$. 
Table 3. Expressions of ZNF436, $\beta$-catenin and CMTM5 in breast cancer.

\begin{tabular}{|c|c|c|c|c|c|c|c|}
\hline \multirow{2}{*}{$\begin{array}{l}\text { Clinicopathological } \\
\text { characteristics }\end{array}$} & \multirow{2}{*}{$\begin{array}{c}\text { Case } \\
\text { number }\end{array}$} & \multicolumn{2}{|c|}{ ZNF436 (positive rate) } & \multicolumn{2}{|c|}{$\beta$-catenin (positive rate) } & \multicolumn{2}{|c|}{ CMTM5 (positive rate) } \\
\hline & & $\begin{array}{l}\text { High- } \\
\text { expression }\end{array}$ & $\begin{array}{l}\text { Low- } \\
\text { expression }\end{array}$ & $\begin{array}{l}\text { High- } \\
\text { expression }\end{array}$ & $\begin{array}{l}\text { Low- } \\
\text { expression }\end{array}$ & $\begin{array}{l}\text { High- } \\
\text { expression }\end{array}$ & $\begin{array}{l}\text { Low- } \\
\text { expression }\end{array}$ \\
\hline \multicolumn{8}{|l|}{ Age } \\
\hline$<55$ & 27 & $25(92.6 \%)$ & $2(7.4 \%)$ & $19(70.4 \%)$ & $8(29.6 \%)$ & $15(55.6 \%)$ & $12(44.4 \%)$ \\
\hline$\geq 55$ & 53 & $47(88.7 \%)$ & $6(11.3 \%)$ & $41(77.4 \%)$ & $12(22.6 \%)$ & $32(60.4 \%)$ & $20(39.6 \%)$ \\
\hline \multicolumn{8}{|l|}{ Tumor size } \\
\hline$<5 \mathrm{~cm}$ & 61 & $55(90.2 \%)$ & $6(9.8 \%)$ & $40(65.6 \%)$ & $21(34.4 \%)$ & $51(83.6 \%)$ & $10(16.4 \%)$ \\
\hline$\geq 5 \mathrm{~cm}$ & 19 & $18(94.7 \%)$ & $1(5.3 \%)$ & $11(57.9 \%)$ & $8(42.1 \%)$ & $12(63.2 \%)$ & $7(36.8 \%)$ \\
\hline \multicolumn{8}{|l|}{ Clinical stages } \\
\hline $\mathrm{I}+\mathrm{II}$ & 30 & $24(80 \%)$ & $6(20 \%)$ & $16(53.3 \%)$ & $14(46.7 \%)$ & $22(73.3 \%)$ & $8(26.7 \%)$ \\
\hline III+IV & 50 & $44(88 \%)$ & $6(12 \%)$ & $31(62 \%)$ & $19(38 \%)$ & $34(68 \%)$ & $16(32 \%)$ \\
\hline \multicolumn{8}{|l|}{ Differentiation } \\
\hline $\begin{array}{l}\text { Highly/moderately } \\
\text { differentiated }\end{array}$ & 55 & $51(92.7 \%)$ & $4(7.3 \%)$ & $30(54.5 \%)$ & $25(45.5 \%)$ & $32(58.2 \%)$ & $23(41.8 \%)$ \\
\hline Poorly differentiated & 25 & $18(72 \%)$ & $7(28 \%)$ & $16(64 \%)$ & $9(36 \%)$ & $20(80 \%)$ & $5(20 \%)$ \\
\hline \multicolumn{8}{|l|}{ Lymphatic metastasis } \\
\hline Yes & 38 & $37(97.4 \%)$ & $1(2.6 \%)$ & $20(52.6 \%)$ & $18(47.4 \%)$ & $26(68.4 \%)$ & $12(31.6 \%)$ \\
\hline No & 42 & $40(95.2 \%)$ & $2(4.8 \%)$ & $24(57.1 \%)$ & $18(42.9 \%)$ & $31(73.8 \%)$ & $11(26.2 \%)$ \\
\hline
\end{tabular}

Table 4. Correlations of ZNF436, $\beta$-catenin and CMTM5 expressions with breast cancer.

\begin{tabular}{|c|c|c|c|c|c|c|c|}
\hline \multirow{2}{*}{$\begin{array}{l}\text { Clinicopathological } \\
\text { characteristics }\end{array}$} & \multirow{2}{*}{$\begin{array}{c}\text { Case } \\
\text { number }\end{array}$} & \multicolumn{2}{|c|}{ ZNF436 (positive rate) } & \multicolumn{2}{|c|}{$\beta$-catenin (positive rate) } & \multicolumn{2}{|c|}{ CMTM5 (positive rate) } \\
\hline & & $\chi^{2}$ & p & $\chi^{2}$ & p & $\chi^{2}$ & p \\
\hline $\begin{array}{l}\text { Age } \\
<55 \\
\geq 55\end{array}$ & $\begin{array}{l}27 \\
53\end{array}$ & 1.448 & 0.632 & 0.486 & 0.881 & 1.552 & 0.674 \\
\hline $\begin{array}{l}\text { Tumor size } \\
\quad<5 \mathrm{~cm} \\
\quad \geq 5 \mathrm{~cm}\end{array}$ & $\begin{array}{l}61 \\
19\end{array}$ & 10.444 & 0.000 & 7.146 & 0.012 & 4.233 & 0.071 \\
\hline $\begin{array}{l}\text { Clinical stages } \\
\text { I+II } \\
\text { III+IV }\end{array}$ & $\begin{array}{l}30 \\
50\end{array}$ & 4.257 & 0.031 & 5.810 & 0.004 & 7.354 & 0.038 \\
\hline $\begin{array}{l}\text { Differentiation } \\
\text { Highly/moderately differentiatec } \\
\text { Poorly differentiated }\end{array}$ & $\begin{array}{l}55 \\
25\end{array}$ & 22.901 & 0.000 & 12.455 & 0.007 & 3.987 & 0.019 \\
\hline $\begin{array}{l}\text { Lymphatic metastasis } \\
\text { Yes } \\
\text { No } \\
\end{array}$ & $\begin{array}{l}38 \\
42 \\
\end{array}$ & 4.585 & 0.05 & 3.278 & 0.019 & 6.338 & 0.042 \\
\hline
\end{tabular}

Table 5. EGFR expression levels in breast cancer and analysis on their correlations with clinicopathological characteristics

\begin{tabular}{|c|c|c|c|c|c|}
\hline \multirow[t]{2}{*}{ Clinicopathological characteristics } & \multirow[t]{2}{*}{ Case number } & \multicolumn{2}{|c|}{ EGFR (positive rate) } & \multirow[t]{2}{*}{$\chi^{2}$ value } & \multirow[t]{2}{*}{ p } \\
\hline & & High-expression & Low-expression & & \\
\hline \multicolumn{6}{|l|}{ Age } \\
\hline$<55$ & 27 & $17(63 \%)$ & $10(37 \%)$ & 3.825 & 0.332 \\
\hline$\geq 55$ & 53 & $45(84.9 \%)$ & $8(15.1 \%)$ & & \\
\hline \multicolumn{6}{|l|}{ Tumor size } \\
\hline$<5 \mathrm{~cm}$ & 61 & $42(68.9 \%)$ & $19(31.1 \%)$ & 1.397 & 0.875 \\
\hline$\geq 5 \mathrm{~cm}$ & 19 & $17(89.5 \%)$ & $2(10.5 \%)$ & & \\
\hline \multicolumn{6}{|l|}{ Clinical stages } \\
\hline$I+I I$ & $24(80 \%)$ & $6(20 \%)$ & 2.072 & 0.049 & \\
\hline III+IV & $33(66 \%)$ & $17(34 \%)$ & & & \\
\hline \multicolumn{6}{|l|}{ Differentiation } \\
\hline Highly/moderately differentiated & 55 & $46(83.6 \%)$ & $9(16.4 \%)$ & 1.996 & 0.028 \\
\hline Poorly differentiated & 25 & $21(84 \%)$ & $4(16 \%)$ & & \\
\hline \multicolumn{6}{|l|}{ Lymphatic metastasis } \\
\hline Yes & 38 & $28(73.7 \%)$ & $10(26.3 \%)$ & 3.528 & 0.007 \\
\hline No & 42 & $33(78.6 \%)$ & $9(21.4 \%)$ & & \\
\hline
\end{tabular}


With the emergence of molecular targeting treatment in recent years, scholars begin to attach increasingly great importance to abnormally expressed molecules in the course of breast cancer occurrence. It is expected that not only can molecular markers of breast cancer be identified at a molecular level, but molecular pathogenesis may be clarified. ${ }^{19}$ In the present study, four genes that may become molecular markers of breast cancer are selected, that is, ZNF436, $\beta$-catenin, EGFR and CMTM5. Variations in their expression levels in breast cancer tissues are analyzed, so are their correlations to clinicopathological characteristics of patients. As revealed by relevant results, ZNF436, $\beta$-catenin and EGFR significantly are highly expressed in breast cancer tissues, while the expression level of CMTM5 there is down-regulated. In the future, they may be used as molecular targets in the treatment and diagnosis of breast cancer.

ZNFs, as important transcription factors, extensively participate in the occurrence and progression of various tumors, playing a part in inhibiting or promoting the cancer. ZNF436 is a critical member of ZNFs. However, tumors involving ZNF436 are seldom investigated, but other transcription factors of this family have been reported to be concerned with various life actions. It has been manifested in lots of research that cell resistance to apoptosis and chemotherapy resistance enhancement, etc. are directly associated with ZNF703 expression upregulation. Alternative splicing isomers ZNF148-FL and ZNF148 4 N of ZNF148 are present. ${ }^{20}$ It has been demonstrated by Liu et al. ${ }^{20}$ that ZNF148-FL has the potential to promote proliferation, invasion and migration of colorectal cancer cells; nevertheless, the opposite is true for ZNF148 $\Delta \mathrm{N}$. The above two alternative splicing isomers of ZNF148 may exert a mutual antagonistic action on bioactivity of malignant tumors. ${ }^{20}$ In an overview presented by Gualtero et al., it is pointed out that ZNF510 in oral cancer is the only newly emerging biomarker in patients at T1+T2 or T3+T4 stages; moreover, ZNF510 shows high sensitivity and specificity in differentiating early and late tumors. ${ }^{21}$ Beyond that, ZNFs are also involved in occurrence and progression of colorectal cancer, head and neck squamous cell carcinoma and stomach cancer, etc. ${ }^{19}$ They may serve as independent indexes for prognosis prediction. ${ }^{22-24}$

$\beta$-catenin, a key member in Wnt/ $\beta$-catenin signaling pathway, is proven to play a regulating role in various diseases, including occurrence and progression of tumors. With high heterogeneity, liver cancer is concerned with regulation on multiple signaling pathways. Wnt $/ \beta$-catenin signaling pathways are frequently upregulated in hepatocellular carcinoma (HCC) and related to tumor maintenance, drug resistance, and tumor progression and metastasis. Therefore, finding an appropriate drug acting on the Wnt $/ \beta$ catenin signaling pathway is of particular significance for $\mathrm{Wnt} / \beta$ catenin treatment. ${ }^{25}$ According to Yang et al., Tankyrase (TNKS) is a carcinogen for ovarian carcinoma cell proliferation. It may promote aerobic glycolysis by activating Wnt/ $\beta$-catenin signaling. This reflects that TNKS may be a potential molecular target for clinical treatment of $\mathrm{Wnt} / \beta$-catenin dependent ovarian cancer. ${ }^{26} \mathrm{As}$ $\mathrm{Wnt} / \beta$-catenin signaling pathway plays a critical role, targeting it or one of its downstream pathways is considered as an effective approach to improve chemotherapeutic efficacy for tumors, contributing to their treatment. ${ }^{27}$

Gene polymorphism of CMTM may influence susceptibility of individuals to cancers. In a survey by Bei et al., how polymorphism of CMTM family genes in the populations in southern China is correlated to HCC is explored. As shown by relevant results, polymorphism of rs3811178 in CMTM5 and rs164207 in CMTM6 may be associated with hereditary susceptibility to HCC among the southern people in China. ${ }^{28}$ Moreover, CMTM5 also has the capability to inhibit proliferation of tumor cells in prostatic cancer, liver cancer and ovarian cancer, promote apoptosis, and lower both invasiveness and migration capability of cells. Therefore, it may be used as a potential target for tumor treatment. ${ }^{29,30}$

In terms of physiological functions, EGFR has a function to regulate development and homeostasis of epithelial tissues. It may turn into a driving factor for tumorigenesis in a pathological context. Through cell cycle disorder, EGFR can promote and activate related signaling pathways, such as $\mathrm{Wnt} / \beta$-catenin mentioned above. Activation induced by EGFR may accelerate occurrence of lung cancer, and head and neck cancer, etc., as well as raise the resistance to drugs. ${ }^{31,32}$ EGFR is not only extensively highlighted in research on tumors, but also critical to tissue repair. ${ }^{32,33}$ In addition, EGFR is proven to be able to repair renal injuries. For example, EGFR can be activated shortly after wounding of renal tubular cells, signifying that EGFR is able to significantly facilitate wound repair. ${ }^{33}$ Severe acute respiratory syndrome coronavirus (SARS$\mathrm{CoV}$ ), a highly pathogenic respiratory virus, can attack human beings and cause death. Infection with this virus may lead to acute lung injuries and pulmonary dysfunction. In this case, not only should lung injuries be repaired, but pulmonary dysfunction needs to be relieved. Pulmonary fibrosis may be developed among many patients infected with SARS-CoV. It is found by Venkataraman et al. that EGFR-regulating wound healing approach is critical to repair of tissue injuries induced by SARS-CoV in a mouse model with an onset of SARS-CoV. In conformity with their research findings, regulating the activation induced by EGFR may prevent fibrosis progression caused by infection with SARS-CoV. ${ }^{34}$ As far as the research made by Shi et al. is concerned, a SD rat is utilized to construct a peptic ulcer model; and the model is further adopted to explore influence of tissue glue on mast cells (MCs) and EGFR in the rat. It turns out that the tissue glue is able to enhance regeneration and repair capability of gastrointestinal mucosa via EGFRmRNA up-regulation, so as to reduce the number of MCs and promote gastrointestinal mucosa healing. ${ }^{35}$ Moreover, EGFR is demonstrated to play an essential role in tissue repair in lots of the existing literature.

The present study, focused on ZNF436, $\beta$-Catenin, EGFR and CMTM5, reveals that they are critical to breast cancer. Hopefully, appropriate molecular targets can be provided for early diagnosis and treatment of breast cancer. It is believed that the clinical application prospect of molecular targeting treatment will become more promising as relevant research becomes increasingly profound.

\section{References}

1. Anastasiadi Z, Lianos GD, Ignatiadou E, Harissis HV, Mitsis M. Breast cancer in young women: an overview. Updates Surg 2017;69:313-7.

2. Bernhard W. Breast cancer: basics, screening, diagnostics, and treatment. Med Monatsschr Pharm 2017;40:55-64.

3. Fahad Ullah M. Breast cancer: Current perspectives on the disease status. Adv Exp Med Biol 2019;1152:51-64.

4. Shang Y, Li Y, Zhang Y, Wang J. ZNF436 promotes tumor cell proliferation through transcriptional activation of BCL10 in glioma. Biochem Biophys Res Commun 2019;515:572-8.

5. Alamoud KA, Kukuruzinska MA. Emerging insights into Wnt $/ \beta$-catenin signaling in head and neck cancer. J Dent Res 2018;97:665-73.

6. Shang S, Hua F, Hu ZW. The regulation of $\beta$-catenin activity and function in cancer: therapeutic opportunities. Oncotarget 2017;8:33972-89.

7. Yang F, Fang E, Mei H, Chen Y, Li H, Li D, et al. Cis-acting circ-CTNNB1 promotes $\beta$-catenin signaling and cancer progression via DDX3-mediated transactivation of YY1. Cancer 
Res 2019;79:557-1.

8. Wang Z. ErbB receptors and cancer. Methods Mol Biol 2017;1652:3-35.

9. Yasuda H, Kobayashi S, Costa DB. EGFR exon 20 insertion mutations in non-small-cell lung cancer: preclinical data and clinical implications. Lancet Oncol 2012;13:e23-e31. Erratum in Lancet Oncol 2011;12:1182.

10. Cai B, Xiao Y, Li Y, Zheng S. CMTM5 inhibits renal cancer cell growth through inducing cell-cycle arrest and apoptosis. Oncol Lett 2017;14:1536-42.

11. Yuan YQ, Zhang YX, Liu ZH, Qin CP, Sheng ZZ, Xu T, et al. [Expression and significance of CMTM5 and epidermal growth factor receptor in prostate cancer].[Article in Chinese]. Beijing Da Xue Xue Bao Yi Xue Ban 2015;47:571-6.

12. Xiao Y, Yuan Y, Zhang Y, Li J, Liu Z, Zhang X, et al. CMTM5 is reduced in prostate cancer and inhibits cancer cell growth in vitro and in vivo. Clin Transl Oncol 2015;17:431-7.

13. Guo X, Li T, Wang Y, Shao L, Zhang Y, Ma D, et al. CMTM5 induces apoptosis of pancreatic cancer cells and has synergistic effects with TNF-alpha. Biochem Biophys Res Commun 2009;387:139-42.

14. Wu J. CMTM5/7 are biomarkers and prognostic factors in human breast carcinoma. Cancer Biomark 2020;29:89-99.

15. Zhou J, Lei J, Wang J, Lian CL, Hua L, He ZY, et al. Bioinformatics-based discovery of CKLF-like MARVEL transmembrane member 5 as a novel biomarker for breast cancer. Front Cell Dev Biol 2020;7:361.

16. Varma K, Chauhan A, Bhargava M, Misra V, Srivastava S. Association of different patterns of expression of beta-catenin and cyclin D1 with pathogenesis of breast carcinoma. Indian J Pathol Microbiol 2020;63:13-8.

17. Song X, Liu Z, Yu Z. EGFR promotes the development of triple negative breast cancer through JAK/STAT3 Signaling. Cancer Manag Res 2020;12:703-17.

18. Hong W, Dong E. The past, present and future of breast cancer research in China. Cancer Lett 2014;351:1-5.

19. Odle TG. Precision medicine in breast cancer. Radiol Technol 2017;88:401M-21.

20. Liu Y, Huang W, Gao X, Kuang F. Regulation between two alternative splicing isoforms ZNF148FL and ZNF148 $\Delta$ N, and their roles in the apoptosis and invasion of colorectal cancer. Pathol Res Pract 2019;215:272-7.

21. Gualtero DF, Suarez Castillo A. Biomarkers in saliva for the detection of oral squamous cell carcinoma and their potential use for early diagnosis: a systematic review. Acta Odontol Scand 2016;74:170-7.

22. O'Reilly JA, Fitzgerald J, Fitzgerald S, Kenny D, Kay EW, O'Kennedy R, et al. Diagnostic potential of zinc finger protein- specific autoantibodies and associated linear B-cell epitopes in colorectal cancer. PLoS One 2015;10:e123469.

23. Gaykalova DA, Vatapalli R, Wei Y, Tsai HL, Wang H, Zhang $\mathrm{C}$, et al. Outlier analysis defines zinc finger gene family DNA methylation in tumors and saliva of head and neck cancer patients. PLoS One 2015;10:e142148.

24. Pan HX, Bai HS, Guo Y, Cheng ZY. Bioinformatic analysis of the prognostic value of ZNF860 in recurrence-free survival and its potential regulative network in gastric cancer. Eur Rev Med Pharmacol Sci 2019;23:162-70.

25. Vilchez V, Turcios L, Marti F, Gedaly R. Targeting Wnt/ $\beta$ catenin pathway in hepatocellular carcinoma treatment. World J Gastroenterol 2016;22:823-32.

26. Yang HY, Shen JX, Wang Y, Liu Y, Shen DY, Quan S. Tankyrase promotes aerobic glycolysis and proliferation of ovarian cancer through activation of $\mathrm{Wnt} / \beta$-catenin signaling. Biomed Res Int 2019;2019:2686340.

27. Emons G, Spitzner M, Reineke S, Möller J, Auslander N, Kramer F, et al. Chemoradiotherapy resistance in colorectal cancer cells is mediated by $\mathrm{Wnt} / \beta$-catenin signaling. Mol Cancer Res 2017;15:1481-90.

28. Bei C, Tan C, Zhu X, Wang Z, Tan S. Association between polymorphisms in CMTM family genes and hepatocellular carcinoma in Guangxi of China. DNA Cell Biol 2018;37:691-6.

29. Xu G, Dang C. CMTM5 is downregulated and suppresses tumour growth in hepatocellular carcinoma through regulating PI3K-AKT signalling. Cancer Cell Int 2017;17:113.

30. Li P, Liu K, Li L, Yang M, Gao W, Feng J, et al. Reduced CMTM5 expression correlates with carcinogenesis in human epithelial ovarian cancer. Int J Gynecol Cancer 2011;21:1248-55.

31. Liu X, Wang P, Zhang C, Ma Z. Epidermal growth factor receptor (EGFR): A rising star in the era of precision medicine of lung cancer. Oncotarget 2017;8:50209-20.

32. Byeon $\mathrm{HK}, \mathrm{Ku} \mathrm{M}$, Yang J. Beyond EGFR inhibition: multilateral combat strategies to stop the progression of head and neck cancer. Exp Mol Med 2019;51:1-14.

33. Zhou X, Zhang W, Yao Q, Zhang H, Dong G, Zhang M, et al. Exosome production and its regulation of EGFR during wound healing in renal tubular cells. Am J Physiol Renal Physiol 2017;312:F963-70.

34. Venkataraman T, Coleman CM, Frieman MB. Overactive epidermal growth factor receptor signaling leads to increased fibrosis after Severe Acute Respiratory Syndrome Coronavirus infection. J Virol 2017;91:e00182-17.

35. Shi YY, Liu HF, Min M, Wang W, Li J, He CY, et al. Correlation analysis of mast cells and EGFR with endoscopic application of tissue glue for treatment of peptic ulcer healing. Eur Rev Med Pharmacol Sci 2017;21:861-6.

Received for publication: 13 August 2020. Accepted for publication: 7 December 2020.

This work is licensed under a Creative Commons Attribution-NonCommercial 4.0 International License (CC BY-NC 4.0).

CCopyright: the Author(s), 2021

Licensee PAGEPress, Italy

European Journal of Histochemistry 2021; 65:3173

doi:10.4081/ejh.2021.3173 